\title{
Über die Umwandlung von Lävulose in Dextrose in der künstlich durchströmten Leber.
}

\author{
Von \\ S. Isaac. \\ Mit 5 Kurvenzeichnungen im Text. \\ (Aus dem chemisch-physiologischen Institut zu Frankfurt a. M.) \\ (Der Redaktion zugegangen am 9. Dezember 1913.)
}

Es ist bekannt, daß zwischen den Aldo- und Ketozuckern der Hexanreihe in chemischer und biologischer Hinsicht enge Beziehungen bestehen. Lobry de Bruyn und Alberda van Eckenstein konnten schon vor längerer Zeit zeigen, daß in vitro unter Einwirkung von Alkalien strukturell zusammengehörige Zucker ineinander umgelagert werden.

In einer kürzlich erschienenen Arbeit haben Embden, Schmitz und Wittenberg ${ }^{1}$ ) die Stellung der Ketozucker zu den Aldehydzuckern von physiologischen Gesichtspunkten aus besprochen. Auf Grund der von ihnen gemachten Feststellung, $\mathrm{da} B$ bei der Durchströmung der überlebenden Leber mit inaktivem Glycerinaldehyd unter Beteiligung der unnatürlichen Komponente desselben d-Sorbose entsteht, äußerten sie die Vorstellung, daß auch die Synthese von $d$-Glukose aus natürlichem Glycerinaldehyd unter intermediärer Bildung einer der Glukose entsprechenden Ketose d. h. von d-Lävulose erfolgen könne. Sie wiesen dabei schon auf das wesentliche Ergebnis der vorliegenden Arbeit hin, daß in der überlebenden Leber eine Umwandlung von d-Lävulose in d-Glukose tatsächlich stattfindet.

1) Embden, Schmitz u. Wittenberg, Über synthetische Zuckerbildung in der künstlich durchströmten Leber, Diese Zeitschrift, Bd. 88, S. 210, 1913. 
Die folgenden Versuche, welche dies zeigen, wurden in der Weise ausgeführt, daß überlebende Hundelebern mit gewaschenen Blutkörperchen unter Lävulosezusatz durchströmt wurden, und durch mehrfache Entnahmen des aus den Lebervenen abfließenden Blutes während der anderthalbstündigen Versuchsdauer die Dextrosebildung aus Lävulose kurvenmäßig verfolgt wurde. Die.Versuchsanordnung entsprach in ihren Einzelheiten der von Embden, Schmitz uud Wittenberg in ihrer eben erwähnten Arbeit ausführlich geschilderten.

Die Bestimmung des in den entnommenen Proben des Durchströmungsblutes jeweils vorhandenen Trauben-und Fruchtzuckers geschah in folgender Weise:

$80 \mathrm{ccm}$ Blut wurden mit kolloidalem Eisenoxyd gefällt. Nach der Fällung betrug die Gesamtflüssigkeitsmenge $1050 \mathrm{ccm}$. $600 \mathrm{ccm}$ des Filtrates wurden im Vakuum eingeengt und auf genau $50 \mathrm{ccm}$ aufgefüllt. Es wurde einerseits das Drehungsvermögen dieser Flüssigkeit im 4-Dezimeterrohr, anderseits ihre Reduktionskraft nach Maquenne bestimmt. Aus dem polarimetrischen und dem Reduktionswert wurde ähnlich, wie dies Tollens ${ }^{1}$ ) angibt, der jeweilige Gehalt des Blutes an Dextrose und Lävulose bestimmt, wobei wir durch Anbringung einer Korrektur auch der geringeren Reduktionskraft der Lävulose Rechnung trugen.

\section{Versuch 1.}

Hund vom Gewicht 7,4 kg erhielt vom 20.9.13 bis 23 . 9.13 täglich dreimal $4 \mathrm{ccm}$ einer $2 \%$ igen alkoholischen Phloridzinlösung. Am 23. 9. Durchblutung mit gewaschenen Rinderblutkörperchen in Ring er-Lösung. MengederDurchströmungsflüssigkeit: $2300 \mathrm{ccm}$. Gewicht der Leber: $253 \mathrm{~g}$. 30 Minuten nach Beginn der Durchblutung Zusatz von 10,4 g Lävulose (aus Inulin, Präparat I von Kahlbaum). Dauer der Durchblutung: 90 Minuteñ:

S. 145.

1) Tollens, Handbuch der biochem. Arbeitsmethoden, Bd. 2, 
Tabelle 1.

\begin{tabular}{|c|c|c|c|c|c|c|c|c|c|}
\hline $\begin{array}{c}\text { Zeitpunkt } \\
\text { nach Beginn der } \\
\text { Durchblutung }\end{array}$ & $10 \mathrm{Min}$. & $20 \mathrm{Min}$. & $30 \mathrm{Min}$. & $40 \mathrm{Min}$. & $50 \mathrm{Min}$. & $60 \mathrm{Min}$ & $70 \mathrm{Min}$ & $80 \mathrm{Min}$ & 90 Min. \\
\hline $\begin{array}{l}\text { In } 30 \text { ccm des ein. } \\
\text { geengen Filtrates } \\
\text { vorhandenc } \\
\text { Gesamtreduktion } \\
\text { in } g\end{array}$ & 0,008 & 0,010 & 0,010 & 0,189 & 0,187 & 0,189 & 0,196 & 0,181 & 0,192 \\
\hline $\begin{array}{l}\text { Drehungswinkel } \\
\text { dieser Flüssigkeit }\end{array}$ & $+0,07^{\circ}$ & $+0,08^{\circ}$ & $+0,08^{\circ}$ & $-1,25^{\circ}$ & $-0,96^{\circ}$ & $-0,72^{\circ}$ & $-0,45^{\circ}$ & $\mid-0,15^{\circ}$ & $+0,11^{\circ}$ \\
\hline $\begin{array}{c}\text { Korrigierter } \\
\text { Reduktionswert } \\
\text { in Prozent des } \\
\text { Durchströmungs- } \\
\text { blutes }\end{array}$ & 0,018 & 0,023 & 0,024 & 0,416 & 0,413 & 0,420 & 0,437 & 0,406 & 0,430 \\
\hline $\begin{array}{l}\text { In } 100 \mathrm{ccm} \text { Durch- } \\
\text { strömungsblut } \\
\text { vorhandene } \\
\text { Dextrose in } \mathrm{g}\end{array}$ & 0018 & 0093 & גרת 0 & 0050 & 0098 & 0134 & 19? 0 & 0929 & 0.295 \\
\hline Lävulose » & - & - & - & $0,36 \tilde{5}$ & 0,314 & 0,286 & 0,235 & 0,172 & 0,135 \\
\hline
\end{tabular}

Versuch 2.

Hund vom Gewicht 9,2 kg erhält vom 28. 9. bis 1.10.13 täglich dreimal $4 \mathrm{ccm}$ einer $2 \%$ igen alkoholischen Phloridzinlösung. Am 1. 10. 13 Durchblutung der $370 \mathrm{~g}$ schweren Leber mit gewaschenen Rinderblutkörperchen in Ringer-Lösung. Menge der Durchströmungsflüssigkeit: $2300 \mathrm{ccm}$. Nach $30 \mathrm{Mi}$ nuten Zusatz von 10,4 g Lävulose in $200 \mathrm{ccm}$ Wasser.

Tabelle 2.

\begin{tabular}{|c|c|c|c|c|c|c|c|c|c|}
\hline $\begin{array}{l}\text { Zeitpunkt nach } \\
\text { Beginn der } \\
\text { Durchblutung }\end{array}$ & $10 \mathrm{Min}$ & $20 \mathrm{Min}$. & 30Min. & $40 \mathrm{Min}$ & $50 \mathrm{Min}$. & $60 \mathrm{Min}$. & 70 Min. & $80 \mathrm{Min}$ & 90 Miń. \\
\hline $\begin{array}{c}\text { In } 50 \mathrm{ccm} \text { Filtrat } \\
\text { festgestellte } \\
\text { Gesamtreduktion } \\
\text { in } \mathrm{g} \\
\end{array}$ & 0,0137 & 0,0141 & 0,0141 & 0,1785 & 0,1740 & 0,1728 & 0,1691 & 0,1746 & 0,1767 \\
\hline $\begin{array}{l}\text { Drehungswinkel } \\
\text { dieser Flüssigkeit }\end{array}$ & $+0,05^{0}$ & $+0,06^{\circ}$ & $+0,06^{\circ}$ & $-0,98^{\circ}$ & $-0,70^{\circ}$ & $-0,48^{\circ}$ & $-0,19^{\circ}$ & $+0,03^{\circ}$ & $+0,29^{\circ}$ \\
\hline $\begin{array}{c}\text { Korrigierter } \\
\text { Reduktionswert } \\
\text { in Prozent des } \\
\text { Durchströmungs- } \\
\text { blutes }\end{array}$ & 0,026 & 0,031 & 0,031 & 0,393 & 0,385 & 0,384 & 0,388 & 0,392 & 0,392 \\
\hline $\begin{array}{l}\text { In } 100 \mathrm{ccm} \text { Durch- } \\
\text { strömungsblut } \\
\text { vorhandene }\end{array}$ & & & & & & & & & \\
\hline Dextrose in $g$ & 0,026 & 0,031 & 0,031 & 0,082 & 0,115 & 0,162 & 0,216 & 0,255 & 0,295 \\
\hline Lävulose in $g$ & - & - & 一 & 0,311 & 0,270 & 0,221 & 0,172 & 0,136 & 0,097 \\
\hline
\end{tabular}


Aus Tabelle 1 ergibt sich also, daß von den 10 Minuten nach Zusatz der Lävulose vorhandenen 0,365\% am Schlusse der Durchblutung noch 0,135\% vorhanden waren; der Dextrosegehalt betrug zu den entsprechenden Zeitpunkten 0,050 und $0,295 \%$. Tabelle 2 zeigt, daß von 0,310\% Lävulose 10 Minuten nach Beginn der Durchblutung, bei Beendigung des Versuches $0,097 \%$ noch vorgefunden wurden. Die entsprechenden Dextrosewerte betrugen in diesem Versuche 0,082 und $0,295 \%$. Daß tatsächlich bei Beendigung dieser beiden Versuche außer der noch geringen Menge von Lävulose nur Dextrose vorhanden war, ergibt sich daraus, daß nach Vergärung keine Drehung und keine Reduktion mehr zu konstatieren war; auch gewannen wir das der d-Glukose und der d-Lävulose gemeinschaftliche d-Glukosazon. Da, wie aus zahlreichen Leerversuchen von Embden, Schmitz und Wittenberg hervorgeht, eine irgendwie erhebliche Zuckerbildung in der glykogenfreien Leber von Phloridzinhunden unter genau den Versuchsbedingungen meiner Arbeit nicht stattindet, bleibt kein anderer Schluß übrig, als daß der nach der Durchblutung vorhandene Traubenzucker einer Umwandlung aus Lävulose seine Entstehung verdankt, zumal die letztere im gleichen Maße abgenommen hat, wie der Dextrosegehalt zunahm.

In gleicher Weise verlief der folgende Versuch, bei dem nicht die Leber eines phloridzindiabetischen Hundes, sondern die eines normalen Hungerhundes zur Durchblutung benutzt wurde.

\section{Versuch 3.}

Hund vom Gewicht 9,5 kg hat 4 Tage gehungert. Gewicht der Leber: $210 \mathrm{~g}$. Durchblutung mit gewaschenen Rinderblutkörperchen. Menge der Durchströmungsflüssigkeit: $2300 \mathrm{ccm}$. Nach 30 Minuten Zusatz von 10,4 $\mathrm{g}$ Lävulose in $200 \mathrm{ccm}$ Wasser zur Durchströmungsflüssigkeit. Gesamtdauer der Durchblutung: 90 Minuten (siehe nebenstende Tabelle).

Aus Tabelle 3 ergibt sich demnach, daß von den 10 Minuten nach Zusatz der Lävulose vorhandenen $0,328 \%$ am Schusse der Durchblutung nur noch 0,063\% vorhanden

Hoppe-Seyler's Zeitschrift f. physiol. Chemie. LXXXIX. 
Tabelle 3 .

\begin{tabular}{|c|c|c|c|c|c|c|c|c|c|}
\hline $\begin{array}{c}\text { Zeitpunkt } \\
\text { nach Beginn der } \\
\text { Durchblutung }\end{array}$ & $10 \mathrm{Min}$. & $20 \mathrm{Min}$. & 30 Min. & 40Min. & $50 \mathrm{Min}$ & $60 \mathrm{Min}$. & 70 Min. & 80 Min. & 90 Min. \\
\hline $\begin{array}{c}\text { In } 50 \text { ccm Filtrat } \\
\text { vorhandene } \\
\text { Gesamtreduktion } \\
\text { in } \mathbf{g}\end{array}$ & 0,0186 & 0,0099 & 0,0149 & 0,1667 & 0,1542 & 0,1446 & 0,1375 & 0,1288 & 0,1263 \\
\hline $\begin{array}{l}\text { Drehungswinkel } \\
\text { dieser Flüssigkeit }\end{array}$ & - & - & $+0,08^{\circ}$ & $-1,40^{\circ}$ & $-0,88^{\circ}$ & $-0,60^{\circ}$ & $-0,51^{\circ}$ & $-0,09^{\circ}$ & $+0,20^{\circ}$ \\
\hline $\begin{array}{c}\text { Korrigierter } \\
\text { Reduktionswert } \\
\text { in Prozent des } \\
\text { Durchströmungs- } \\
\text { blutes }\end{array}$ & 0,0408 & 0,021 & 0,032 & 0,366 & 0,339 & 0,320 & 0,305 & 0,288 & 0,278 \\
\hline $\begin{array}{l}\text { In } 100 \mathrm{ccm} \text { Durch- } \\
\text { strömungsblut } \\
\text { vorhandene }\end{array}$ & & & & & & & & & \\
\hline Dextrose in $g$ & 0,0408 & 0,021 & 0,032 & 0,037 & 0,065 & 0,101 & 0,107 & 0,168 & 0,215 \\
\hline Lävulose » & - & - & - & 0,328 & 0,274 & 0,219 & 0,197 & 0,119 & 0,063 \\
\hline
\end{tabular}

waren; der Dextrosegehalt betrug zu den entsprechenden Zeitpunkten 0,037 und $0,215 \%$.

War in den drei bisherigen Versuchen die Umwandlung der Lävulose zu Dextrose schon in ganz außerordentlichem Maße vor sich gegangen, so sollte im folgenden Versuch festgestellt werden, ob es gelingt, bei noch längerer Ausdehnung des Versuchs, die zugesetzte Lävulose ganz zum Verschwinden zu bringen.

\section{Versuch 4:}

Hund vom Gewicht $6,8 \mathrm{~kg}$ erhält vom 4.10. bis 8.10 .13 dreimal täglich $4 \mathrm{ccm}$ einer $2 \%$ igen alkoholischen Phloridzinlösung. Am 8.10. 13 Durchblutung der $206 \mathrm{~g}$ schweren Leber mit gewaschenen Rinderblutkörperchen in Ringer-Lösung. Menge der Durchströmungsflüssigkeit: $2300 \mathrm{ccm}$. Zu letzterer werden 3 Minuten vor Beginn des Versuches $12 \mathrm{~g}$. Lävulose in $220 \mathrm{ccm}$ Wasser zugesetzt (siehe folgende Tabelle).

Aus Tabelle 4 geht hervor, daß anderthalb Stunden nach Zusatz der Lävulose in der Tat nur noch ganz minimale Mengen derselben vorhanden sind. Der ursprüngliche Lävulose- 
Tabelle 4.

\begin{tabular}{|c|c|c|c|c|c|c|c|c|c|c|}
\hline $\begin{array}{c}\text { Zeitpunkt nach } \\
\text { Beginn der } \\
\text { Durchblutung }\end{array}$ & $\begin{array}{c}\text { Bei } \\
\text { Beginn }\end{array}$ & $\begin{array}{l}\text { Nach } \\
10 \mathrm{Min} .\end{array}$ & $20 \mathrm{Min}$. & $30 \mathrm{Min}$. & 40Min. & $50 \mathrm{Min}$. & $60 \mathrm{Min}$. & 70Min. & $80 \mathrm{Min}$ & $90 \mathrm{Min}$. \\
\hline $\begin{array}{c}\text { In } 50 \mathrm{ccm} \text { des ein- } \\
\text { geengten Filtrats } \\
\text { vorhandene } \\
\text { Gesamtreduktion } \\
\text { in } \mathbf{g}\end{array}$ & 0,159 & 0,169 & 0,161 & 0,154 & 0,152 & 0,155 & $.0,148$ & 0,145 & 0,150 & 0,150 \\
\hline $\begin{array}{c}\text { Drehungswinkel } \\
\text { dieser Flüssigkeit }\end{array}$ & $-1,34^{0}$ & $-1,04^{0}$ & $-0,75^{\circ}$ & $-0,50^{\circ}$ & $-0,21^{0}$ & $+0,01^{\circ}$ & $+0,12^{\circ}$ & $+0,31^{\circ}$ & $+0,46^{\circ}$ & $+0,53^{\circ}$ \\
\hline $\begin{array}{c}\text { Korrigierter } \\
\text { Reduktionswert } \\
\text { in Prozent des } \\
\text { Durchströmungs- } \\
\text { blutes }\end{array}$ & 0,361 & 0,373 & 0,356 & 0,343 & 0,341 & 0,350 & 0,331 & 0,322 & 0,331 & 0,330 \\
\hline $\begin{array}{l}\text { In } 100 \mathrm{ccm} \text { Durch- } \\
\text { strömungsblut } \\
\text { vorhandene }\end{array}$ & & & & & & & & & & \\
\hline Dextrose in $\mathrm{g}$ & 0,012 & 0,058 & 0,098 & 0,132 & 0,181 & 0,225 & 0,234 & 0,263 & 0,297 & 0,310 \\
\hline Lävulose , & 0,349 & 0,315 & 0,258 & 0,211 & 0,159 & 0,125 & 0,097 & 0,059 & 0,034 & 0,020 \\
\hline
\end{tabular}

gehalt von $0,349 \%$ war am Schlusse des Versuchs auf $0,020 \%$ zurückgegangen; die Zunahme der Dextrose war entsprechend.

Schließlich sei noch ein Versuch erwähnt, der aus einer größeren Reihe von mir ausgeführter, noch nicht veröffentlichter Durchblutungsversuche der Lebern phosphorvergifteter Tiere stammt, und der das Verhalten der Lävulose bei der Durchströmung der überlebenden Phosphorleber demonstriert.

Versuch 5:

Hund vom Gewicht $8,8 \mathrm{~kg}$ erhielt an mehreren Tagen insgesamt $0,06 \mathrm{~g}$ Phosphor. Am 14. 8. 13 Tötung des Tieres durch Entbluten, Femoralarterien. Zuckergehalt des Entblutungsblutes: 0,025\%. Gewicht der Leber: $350 \mathrm{~g}$. Die Anordnung der Durchblutung wich von den vorherigen Versuchen insofern $\mathrm{ab}$, als mit $2000 \mathrm{ccm}$ genuinen Rinderblutes durchströmt und die Enteiweißung des Durchströmungsblutes nach Schenck vorgenommen wurde. Das unverdünnte vom Quecksilber befreite Filtrat wurde im $4 \mathrm{dm}$-Rohr polarisiert; in $40 \mathrm{ccm}$ des Filtrats wurde der Zucker nach Maquenne bestimmt. Zusatz von $10 \mathrm{~g}$ Lävulose in Ringer-Lösung 5 Minuten nach Beginn der Durchblutung. 
Tabelle 5 .

\begin{tabular}{c|c|c|c|c}
\hline $\begin{array}{c}\text { Zeitpunkt nach Beginn der } \\
\text { Durchblutung }\end{array}$ & 14 Min. & 31 Min. & 49 Min. & 66 Min. \\
\hline $\begin{array}{c}\text { In } 100 \text { ccm Filtrat festgestellte } \\
\text { Gesamtreduktion in } \mathrm{g}\end{array}$ & 0,069 & 0,060 & 0,057 & 0,057 \\
\hline $\begin{array}{c}\text { Drehungswinkel dieser Flüssig- } \\
\text { keit }\end{array}$ & $-0,25^{\circ}$ & $-0,10^{\circ}$ & $\pm 0^{\circ}$ & $+0,06^{\circ}$ \\
\hline $\begin{array}{c}\text { Korrigierter Reduktionswert } \\
\text { in Prozent des Durchströmungs- } \\
\text { blutes in g }\end{array}$ & 0,418 & 0,369 & 0,352 & 0,347 \\
\hline $\begin{array}{c}\text { In } 100 \text { ccm Durchströmungsblut } \\
\text { vorhandene Dextrose in g }\end{array}$ & 0,026 & 0,140 & 0,225 & 0,289 \\
Lävulose , . & 0,392 & 0,229 & 0,127 & 0,058
\end{tabular}

Aus diesem Versuche ergibt sich die Tatsache, daß auch die bezüglich ihres Kohlenhydratstoffwechsels schwer geschädigte Phosphorleber noch imstande ist, die Umwandlung von Lävulose in Dextrose zu vollziehen.

Auf den nebenstehenden Kurven haben wir das Ergebnis unserer 5 Versuche graphisch dargestellt. Aus allen ergibt sich übereinstimmend, daß mit dem Verschwinden der Lävulose die Dextrose zunimmt. In Versuch 3 (normale Leber) und Versuch 5 (Phosphorleber) ist die Abnahme der Lävulose stärker als die Zunahme der Dextrose. Die Kurve des Gesamtzuckergehaltes zeigt in diesen beiden Fällen eine deutliche Senkung, woraus hervorgeht, daß ein Teil der Lävulose verbraucht wurde, und zwar sicherlich, wie wir aus den Versuchen von S. Oppenheime ${ }^{1}$ ) wissen, unter Bildung von d-Milchsäure. Auch für den speziellen Fall des Versuches 5 zeigten Milchsäurebestimmungen, daß auch die phosphorvergiftete Leber aus Lävulose reichlich Milchsäure bildet. In einem gewissen Gegensatz dazu steht das Verhalten der durchbluteten Lebern der phloridzindiabetischen Hunde, die, wie sich aus den Kurven 1, 2 und 4 ergibt, keine irgendwie beträchtliche Abnahme des Gesamtzuckergehaltes bewirkten, sondern nur

1) S. Oppenheimer, Über Milchsäurebildung in der künstlich durchströmten Leber, Biochem. Zeitschr., Bd. 45, S. 37. 


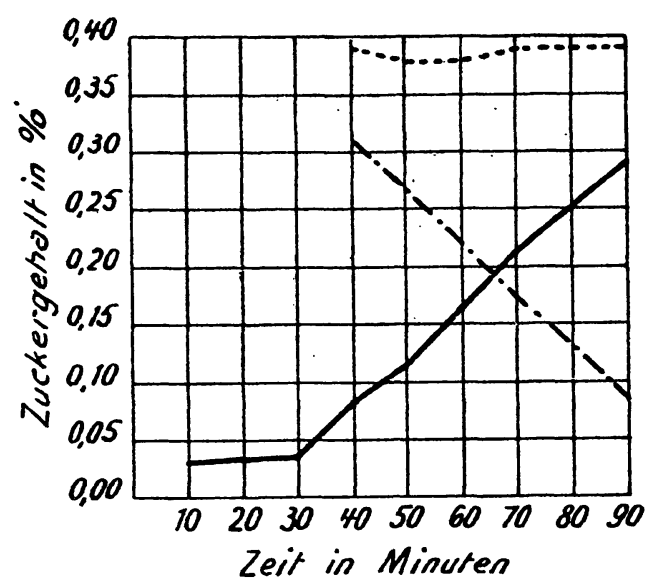

Figur 1.
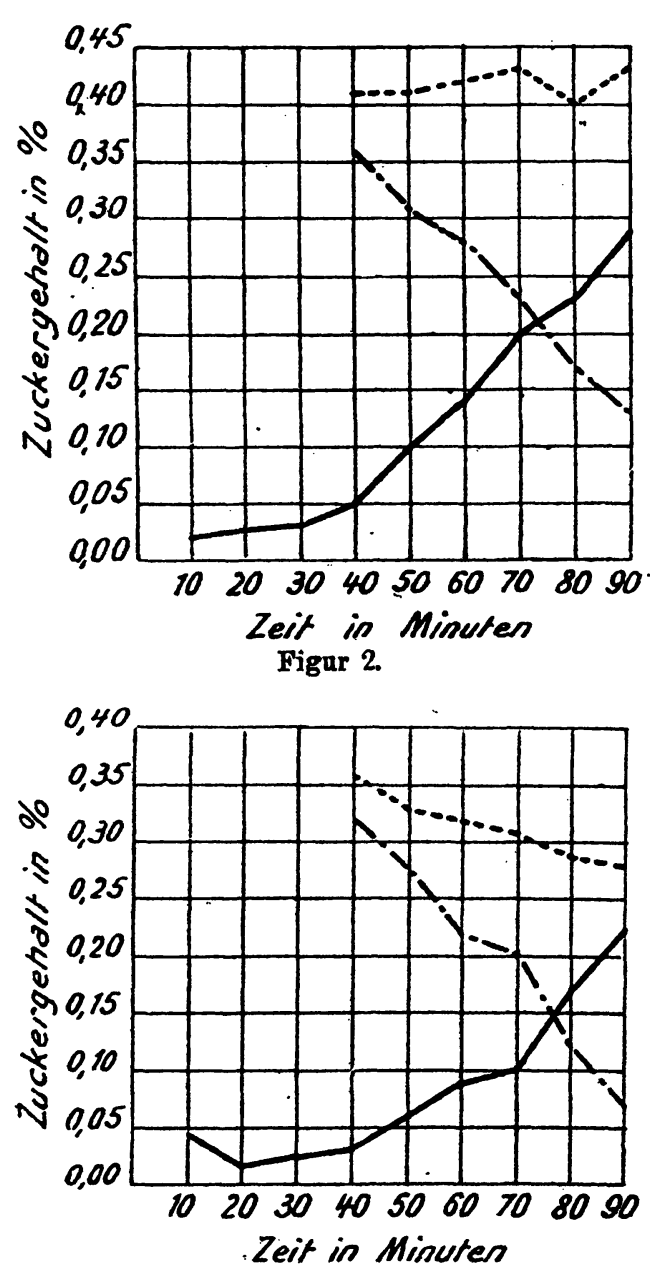

Figur 3.

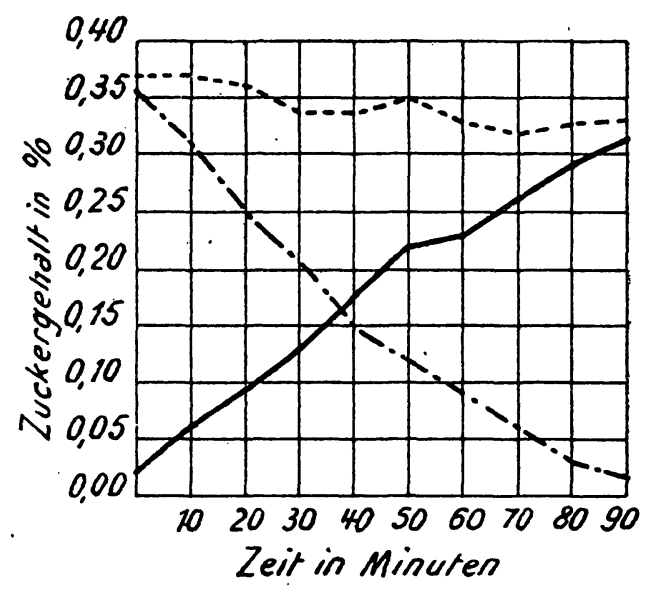

Figur 4.

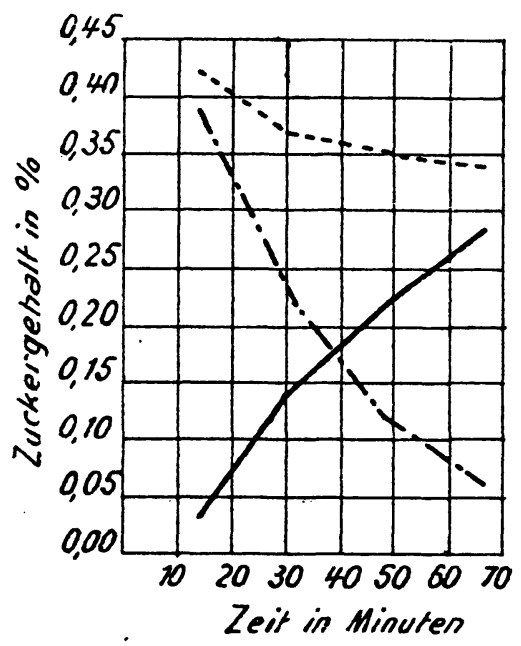

Figur 5. 
die Umwandlung der Lävulose in Dextrose vollzogen. Auf diese Eigentümlichkeit der diabetischen Lebern, die in Hinsicht auf die Theorie des Diabetes vielleicht nicht ohne Bedeutung ist, wird in einer späteren Arbeit näher eingegangen werden.

Ebenso soll in weiteren Versuchen noch besonders festgestellt werden, ob etwa auch andere Organe (Blut, Muskeln, Nieren) Lävulose in Dextrose umlagern können. Wir halten eine ganz allgemeine Verbreitung dieser Reaktion a priori nicht für wahrscheinlich, glauben vielmehr, daß diese Fähigkeit im wesentlichen auf die Leber beschränkt ist.

Vielleicht entsteht während der Umwandlung der Lävulose zu Dextrose in der Leber eine leicht angreifbare Zwischenform, etwa die Enolform. Dadurch würde auch der von S. Oppenheimer ${ }^{1}$ ) festgestellte, im Verhältnis zu Dextrose ungleich leichtere Abbau der Lävulose in der überlebenden Leber verständlich werden. Auch die früher schon von verschiedenen Autoren und neuerdings von Benedikt²) bei Respirationsversuchen gemachte Beobachtung, daß nach Zufuhr von Lävulose eine viel größere Steigerung der $\mathrm{CO}_{2}$-Produktion eintritt, als nach Dextrosefütterung, kann im Sinne des Auftretens einer besonders leicht verbrennbaren Zwischenstufe bei der Umlagerung der Lävulose in Dextrose gedeutet werden. Anderseits spricht der von Rona und $\mathrm{Neukirch}^{3}$ ) erhobene Befund, daß der Herzmuskel sowie die Darmmuskulatur des Kaninchens Lävulose nicht angreifen können, und weiterhin die von Griesbach und Oppenheimer ${ }^{4}$ ) gemachte Feststellung, daß Blutkörperchen im Gegensatz zur Leber Lävulose eher schechter als Dextrose zu Milchsäure abbauen, dafür, daß in diesen Fällen eine Umlagerung der Lävulose zu Dextrose wohl nicht stattfindet.

Die Vollständigkeit und Schnelligkeit, mit der letztere aber in der Leber vor sich geht, darf in der Tat dafür sprechen, daß wir es hier mit einem für die Leberzelle durchaus physiologischen Vorgang zu tun haben. Im Gegensatz zur Alkaliwirkung auf Lävulose in vitro, bei der nach Erreichung des

1) 1. c.

2) Deutsch. Arch. f. klin. Med., Bd. 110.

3) Pflügers Arch., Bd. 144, 146, 148, 1912-13.

4) Biochem. Zeitschr., Bd. 55, 1913. 
Gleichgewichts ein Gemisch von Lävulose, Dextrose und Mannose vorhanden ist, führt die biologische Umwandlung von Lävulose in der Leber nur zum Auftreten von d-Glukose und diese Umlagerung erfolgt schon unter den sicherlich nicht vollkommenen Bedingungen des Durchblutungsversuches in kürzester Zeit und nahezu quantitativ.

Diese Erkenntnis vermittelt uns ein besseres Verständnis mancher bis jetzt noch nicht recht geklärter Einzelheiten des Fruktosestoffwechsels. Dafür, daß Lävulose auch im lebenden Organismus in Dextrose umgewandelt werden kann, sprach schon immer eine Reihe von Tatsachen: Minkowski hatte gezeigt, daß der pankreasdiabetische Hund eingeführte Lävulose zum größten Teil als Dextrose ausscheidet; Bohland, Socin, v. Noorden, Umber u. a. beobachteten beim diabetischen Menschen nach Lävulosezufuhr eine Steigerung der Dextroseausscheidung. Wohl die meisten Autoren schlossen sich der von Voit geäußerten Vorstellung an, daß die nach Einführung von Lävulose auf Kosten dieser gebildete Glykose auf dem Umwege über Glykogen entstehen müsse. Wir glauben aber nach dem Ausfall unserer obigen Versuche auch für diese Fälle eine direkte Umwandlung annehmen $\mathrm{zu}$ müssen und meinen, daß das nach Lävulosedarreichung zur Ablagerung kommende Glykogen erst nach oder während der Umlagerung zu Dextrose gebildet wird. Mit dieser Anschauung stimmt gut überein, daß chemische Differenzen zwischen «dextrogenem» und sfruktogenem. Glykogen niemals gefunden wurden.

Immerhin läßt sich nicht bestreiten, daß nach Verfütterung von Lävulose bezüglich der Glykogenie wenigstens unter pathologischen Verhältnissen bemerkenswerte Unterschiede gegenüber der Dextrose beobachtet werden. Der pankreasdiabetische Hund ist nämlich, wie seit den Untersuchungen Minkowskis bekannt, befähigt, aus Lävulose unter Umständen noch ansehnliche Mengen von Glykogen zu bilden. ${ }^{1}$ ) Und

1) Anmerk. Nach Abschluß dieser Arbeit erschien eine Mitteilung von E. W. H. Cruickshank (Journ. of. Physiol., Bd. 47, S. 1) der nach Lävulosedarreichung beim pankreasdiabetischen Hund keine Glykogenanlagerung in der Leber erzielen konnte, wenn die Exstirpation total war. 
E. Neubauer ${ }^{1}$ ) hat gezeigt, daß sich phosphorvergiftete Kaninchen ganz ähnlich verhalten, wie diabetische Hunde: es finden sich bei ihnen nach Dextrosezufuhr in der Leber nur Spuren, nach Lävulosedarreichung beträchtliche Quantitäten von Glykogen. Diese bisher schwer zu deutenden Befunde lassen sich relativ zwanglos erklären durch die Annahme, daß die aus Lävulose gleichsam in statu nascendi entstehende Dextrose von der diabetischen Leber leichter als Glykogen fixiert werden kann, als der in größerer Menge ohne weitere Vorbereitung mit den Leberzellen in Berührung kommende Traubenzucker. Vielleicht ist aber auch die hypothetische intermediär auftretende, der Dextrose und Lävulose gemeinschaftliche Enolform besonders zur Glykogenbildung geeignet. Damit fallen alle prinzipiellen Unterschiede, die man für die Glykogenbildung aus Lävulose einerseits, aus Dextrose anderseits konstruiert hat, fort, und die Glykogenbildung aus Lävulose beim schweren Diabetes besagt tatsächlich nichts anderes, als daß auch hier unter Bedingungen, welche für die Leberzellen besonders günstig sind, noch eine Glykogenbildung aus Dextrose erfolgen kann.

In einem auffallenden Gegensatz zu dem eben geschilderten Verhalten der Diabetiker zur Lävulose steht die verminderte Lävulosetoleranz der durch irgendwelche Krankheitsprozesse in ihren Funktionen geschädigten Leber, eine Tatsache, die seit ihrer Entdeckung durch Strauß und Sachs immer ein erhebliches theoretisches und praktisches Interesse beansprucht hat und allgemein so interpretiert wird, daß das Glykogenbildungsvermögen aus Lävulose hier Schaden gelitten hat, was nach dem oben mit Hinsicht auf den Diabetes Gesagten schwer verständlich ist. Die Schwierigkeiten der Erklärung werden aber sofort geringer, wenn wir auf Grund unserer Versuche annehmen, daß die Umlagerung der Lävulose zu Dextrose, die wir für eine mehr oder weniger spezifische Funktion der Leber halten, nicht mehr restlos vonstatten geht, sobald größere Mengen in kurzer Zeit an die Leberzellen herantreten, wie es bei der Prüfung auf alimentäre Lävulosurie der Fall ist. Letztere würde also in der Tat eine qualitativ eigenartige

1) Arch. f. exp. Path. u. Pharm., Bd. 61, S. 174. 
Störung der Leberfunktion darstellen, die anders als die Glykosurie bezw. Hyperglykämie nach Dextrosezufuhr zu bewerten ist. Es mag nun auffallen, daß in unserem Versuche 5, dem ich andere gleichartige an die Seite stellen könnte, die Leber des phosphorvergifteten Tieres keine Schädigung des Umlagerungsvermögens zeigte, trotzdem dies beim phosphorvergifteten Tier in vivo in ausgesprochenem Maße der Fall ist. Dies divergente Verhalten ist wohl in der besonderen Art der Versuchsanordnung begründet, insofern eine bestehende Unterwertigkeit der Leber hinsichtlich der Lävuloseumwandlung dadurch ausgeglichen wird, daß die Leberzellen während der künstlichen Durchblutung anders als im lebenden Organismus, wo der im Blute kreisende Lävuloseüberschuß durch die Nieren eliminiert wird, fortwährend aufs intensivste mit der Lävulose in Berührung bleiben.

Wir können somit dạs Ergebnis unserer Versuche dahin zusammenfassen, daß Lävulose mit großer Geschwindigkeit in der künstlich durchströmten Leber zu Dextrose umgewandelt wird. Hierdurch läßt sich eine Reihe von Erscheinungen des Lävulosestoffwechsels relativ zwanglos erklären. Auch die in ihrer eingangs erwähnten Arbeit von Embden, Schmitz und Wittenbèrg ausgesprochene Ansicht, daß bei der biologischen Synthese des Traubenzuckers die Lävulose ein intermediäres Produkt darstellen könne, findet damit eine wesentliche Stütze. Für die Berechtigung einer solchen Anschauung sprechen noch die seltenen von Külz, Schlesinger, Lepine, Boulud, Schwarz, Neubaueri) Strouse und Friedman ${ }^{2}$ ) beschriebenen Fälle von spontaner reiner Fruktosurie. Wohl am eingehendsten hat Neubauer einen derartigen Fall studiert und festgestellt, daß bei seinen Patienten nicht durch kohlenhydratreiche Nahrung oder Traubenzucker, wohl aber durch Lävulose die Fruktoseausscheidung vermehrt wurde.

1) O. Neubauer, Zur Kenntnis der Fruktosurie, Münch. med. W., 1905, S. 1525. (Hier auch die übrige Literatur.)

2) Über Lävulosurie, mit Schilderung eines ungewöhnlichen Falles, Arch. of intern. med., Bd. 9, S. 99, 1912; zit. nach Centralbl. f. d. ges. innere Mediz., Bd. 1, S. 180. 
Fast genau wie der Neubauersche Fall verhielt sich der kürzlich von Strouse und Friedman mitgeteilte. Auch hier war neben der Lävulose niemals Dextrose im Harn nachweisbar. Bei Kohlenhydratentziehung sistierte die Lävuloseausscheidung, sie zeigte aber nach Lärulosezufuhr einen Anstieg, der $10 \%$ der eingeführten Lävulose entsprach, während Dextrosezufuhr die Lävulosurie nicht beeinflußte. Eine befriedigende Erklärung dieser seltenen Fälle stand bis jetzt noch gänzlich aus : wir möchten auch für sie in Analogie mit der alimentären Lävulosurie annehmen, daß hier eine Störung der Umwandlung der intermediär gebildeten Lävulose zu Dextrose vorliegt.

Auch für die einwandsfreien ${ }^{1}$ ) Fälle von Diabetes, wo neben Dextrose Lävulose im Harn erscheint, dürfte ein ähnliches Verhalten zutreffen.

1) Vgl. hierzu H. Königsfeld, Zur Klinik und Pathogenese der Lävulosurie beim Diabetes mellitus, Zeitschr. für klin. Mediz., Bd. 69, S. 291, 1910. 